\title{
Degradation of Rutin into Isoquercitrin by Bacillus litoralis strain C44
}

\author{
Zhitang $\mathrm{Lu}^{1 *}$, Jinglu Wang ${ }^{1}$, Shengxun Lin $^{1}$, Yating Zhang ${ }^{1}$ \\ ${ }^{1}$ College of Life Sciences, Hebei University, Key Laboratory of Microbial Diversity Research and Application of Hebei \\ Province, Baoding, 071002, People's Republic of China
}

\begin{abstract}
To develop the technique of microbial degradation of rutin into isoquercitrin (quercetin 3-glucoside), bacterial strains that could hydrolyze rutin were isolated from Chinese scholar tree Sophora japonica L. soil samples. Strain 44 containing rhanmosidase with the highest transformation ability was obtained after the soil samples were isolated, screened by TLC and re-screened by HPLC. Experiments show that its transformation rate was $50.36 \%$ within a $4 \mathrm{~mL}$ reaction system containing $50 \mathrm{mg}$ wet cells and $0.1 \%$ rutin after transforming for $72 \mathrm{hr}$ at $25^{\circ} \mathrm{C}$. Strain C44 was identified as Bacillus litoralis by integrating the 16S rRNA phylogeny analysis result and a serious of morphological, physiological and biochemical features. The rhanmosidase of strain $\mathrm{C} 44$ is a kind of endoenzyme, and the optimal condition of enzyme reaction is $37^{\circ} \mathrm{C}$ and $\mathrm{pH}$ 7.5.
\end{abstract}

Key words: Rutin; Isoquercitrin; Transformation; Identification; Rhanmosidase

\section{INTRODUCTION}

Rutin, a glycoside composed of quercetin and the disaccharide rutinose, exists in relatively large amounts in bracken ferns, red grapes, buckwheat, apple, and various teas (Kreft et al. 1999; Kuntic et al. 2007). And it is also the major effective components of Flos sophorae, the dried flowers or buds of Chinese scholar tree Sophora japonica L., in which the quantity of rutin is as high as $24.46 \%$ (w/w) (Cheng et al., 2004).

Isoquercitrin is a kind of flavonoid widely distributed in plantage. As a derivative of rutin, the structural difference between them is only a rhanmosidase (Wang et al. 2004). Related to rutin, isoquercitrin has been found to increase blood flow and perhaps be a cure for such maladies as varicose veins, hemorrhoids, and possible use for arterial flow as well. It also has been shown to have anti-irritation properties as well. Many studies have shown its possibilities in increased brain functions due to increased blood flow and might be useful in the treatment of progressive Alzheimer's disease (Dolf De Rovira, 2008). While a certain experiment has showed that rutin has no exhibit anticancer activity (Shen et al., 2003).

The current study also proved that isoquercitrin due to its specific property act as antioxidant, it has an important function in the human body aging and diseases with a high pharmacological activity than rutin (Wang et al., 2004). Besides that, isoquercitrin works better than rutin both in protecting injured cells by scavenging free radical generated from H202 and anoxia/hypoglycemia in cell culture liquid and in inhibiting free radical induced cerebralinjury in mice subjected to cerebral ischemia and hepatic injury in mice subjected to $\mathrm{CCl}_{4}$. (Jin et al. 2006; Jin et al. 2007). And it has a regulative role in blood glucose level and lipids, and improved the function of pancreatic islets (Zhang et al. 2011).

Although isoquercitrin has so many important and special biological activities, its content in nature is extremely low. It is said that the content is only one over ten thousand or even one over more than ten thousand (Yang et al., 1996). Therefore, the study of synthesis and extraction of isoquercitrin, and to find a more efficiency way to obtain quantity isoquercitrin seem to have a vital significance.

Isoquercitrin is previously carried out by chemical and enzymatic methods. In fact, the synthetic procedures are normally tedious and complex, and the overall yields are generally very low (Li et al. 2004). Experiment showed that the aglycon of rutin was deglycosolated easily under mild acidic hydrolysis conditions at appropriate temperatures, but its secondary glucoside was difficult to be obtained. So the prepared isoquercitrin by enzymatic hydrolysis of rutin was preferable to the 
acidic hydrolysis (Wang et al. 2011). Besides, enzymatic catalysis that removes rhamnose from rutinosides also can improve the production of flavonoid glycosides (H’elder Vila-Real et al. 2011).

In the present study, a potential bacterial strain with the ability of producing novel type of rutinosidase was obtained. The enzyme was determined to be rhamnosidase, which has a high efficiency of transforming rutin to isoquercitrin. It can provide a new method for the production of isoquercitrin in the industry.

\section{MATERIALS AND METHODS}

\subsection{Samples}

The soil sample used in this study was collected from the area covered with deciduous Flos Sophorae in Hebei University campus.

\subsection{Medium and reagents}

The medium used for isolating and cultivating was Beef extract-peptone medium, and the pH value is 7.0. Standard rutin and isoquercitrin were purchased from Chengdu Must Bio-Technology Co., Ltd.

\subsection{Isolation of potential isoquercitrin-producing bacteria}

Suspend $10 \mathrm{~g}$ soil sample in sterile water with 20 glass beades, and regulate its $\mathrm{pH}$ to 7.0. The soil sample was incubated at $25^{\circ} \mathrm{C}$ for $25 \mathrm{~min}$. Isoquercitrin-producing bacteria were isolated using serial dilution method by spread suitable dilutions on Beef extract-peptone medium supplemented with $0.1 \%(\mathrm{w} / \mathrm{w})$ rutin. Bacterium-like colonies were inoculated after incubating for $1 \mathrm{~d}$ at $25{ }^{\circ} \mathrm{C}$, and those colonies which change into deep-colored hydrolysis zones by spraying $1.0 \% \mathrm{AlCl}_{3}$-ethanol solution were selected as potential isoquercitrin-producing strains.

\subsection{Screening methods}

\subsubsection{First screening program}

The active strains were first screened by thin layer chromatography (TLC) method, and isolated by culturing in $4 \mathrm{~mL}$ of Beef extract-peptone broth $(\mathrm{pH} 7.0)$ in $15 \times 150 \mathrm{~mm}$ test tubes at $25^{\circ} \mathrm{C}$ in a thermostat shaker with shaking at $280 \mathrm{r} / \mathrm{min}$ for $1 \mathrm{~d}$. Through centrifuging at $4500 \mathrm{r} / \mathrm{min}$ for $5 \mathrm{~min}$ at room temperature $\left(24^{\circ} \mathrm{C} \pm 1{ }^{\circ} \mathrm{C}\right)$, the biomass and the supernatant were separated. To harvest the biomass, the cell pellet was washed with $2 \mathrm{~mL}$ of $0.1 \mathrm{~mol} / \mathrm{L}$ phosphate buffer ( $\mathrm{pH} 7.0$ ) and centrifuged again. After using $4 \mathrm{~mL}$ phosphate buffer (pH7.0) to suspend the bacteria biomass, $4 \mathrm{~mL}$ of cell suspension and the fermentation supernatant were supplemented with $0.1 \%$ rutin and react for $3 \mathrm{~d}$ by shaking at $280 \mathrm{r} / \mathrm{min}$ and $25^{\circ} \mathrm{C}$, repetitively. Finally, TLC analysis was performed to check the ability of the strains in transforming rutin to isoquercitrin.

According to the previous study, the TLC analysis was carried out by loaded the sample on polyamide film and developed with 75\% microemulsion consisted of SDS/n-butyl alcohol/n-heptane/water, 0.27: 0.63: 0.10: 36 (m/m) (Kang et al., 2000). And the developed polyamide film was stained by spraying $1 \% \mathrm{AlCl}_{3}$-ethanol solution and dried under room temperature. TLC polyamide film were scanned and analyzed by UN-scan-it gel scanning software (SIM International Group Co. LTD. U.S.A.).

\subsubsection{Re-screening program}

The active strains were re-screened by High Performance Liquid Chromatography (HPLC), and the retained time of converted products was compared with the one of isoquercitrin standard. The conditions of HPLC were as following: separated by pre-packed Hypersil ODS (C18) column $\left(250 \times 4.6 \mathrm{~mm}\right.$, Elite, China) column at $24^{\circ} \mathrm{C} \pm 1^{\circ} \mathrm{C}$ and detected by UV detector at 360nm, the mobile phase composed of acetonitrile: methanol: water: phosphoric acid (100:10:340:0.3, v/v) was used as the mobile phase at a flow rate of $1.0 \mathrm{~mL} / \mathrm{min}$, sampling volume was $20 \mu \mathrm{L}$ (Jia et al., 2008).

The first step to prepare the HPLC sample is concentrating $4 \mathrm{~mL}$ reaction liquid to $200 \mu \mathrm{L}$ at $40^{\circ} \mathrm{C}$. Then supplement $300 \mu \mathrm{L}$ methanol to it. The precipitation was wiped off after being centrifuged at $4500 \mathrm{r} / \mathrm{min}$ for 5 min. Finally $500 \mu \mathrm{L}$ reaction liquid was got in total. 


\subsection{Determination of transformation rate}

$4.90 \mathrm{mg}$ rutin and $4.90 \mathrm{mg}$ isoquercetin were used for preparing the diluted standards solution of rutin and isoquercitrin, which were used for preparation of different working standards using methanol. $20 \mu \mathrm{L}$ standard solution of each concentration was analyzed by HPLC. The duple gradient concentrations of standard rutin and isoquercitrin were from $0.272 \mu \mathrm{g} / \mathrm{mL}$ to $4.356 \mu \mathrm{g} / \mathrm{mL}$, respectively.

Standard curves were manufactured with the concentration of standard solutions as abscissa and the average peak area of three times as ordinate. The average peak area of the sample was obtained from the liquid chromatogram after three injection times, and the concentration of isoquercitrin was figured out through the standard curve of isoquercitrin and the substrate concentration.

\subsection{Identification of isoquercitrin-producing bacterium strain $\mathrm{C} 44$}

The active bacterium strain C44 was identified by the phylogenetic approaches based on 16S rRNA phylogeney analysis and a series characteristic of morphology, physiology and biochemistry. The genomic DNA of tested strain was extracted with phenol-chloroform method (Marmur, 1961), 16S rDNA was amplified by polymerase chain reaction using universal forward primer 27F (5'-AGAGTTTGATCMTGGCT CAG-3') and reverse primer 1525R (5'-AGAAAGGAGGTGWT CCARCC-3') (Lane, 1999) with described procedure (Lu et al., 2001). The products purified by PCR were directly sequenced by Beijing Sunbiotech Corporation. The obtained sequence was initially estimated by the BLAST facility of NCBI (www.ncbi.nlm.nih.gov/BLAST) and then aligned with all related sequences obtained from GenBank by BioEdit (Hall, 1999). Evolutionary distance matrices were calculated by using the method of Kimura 2-parameter and a neighbour-joining tree was reconstructed by the Mega 5.1 program (Saitou \& Nei, 1987, Tamura et al., 2011).

A light microscopy (Olympus, BH-2) was used to observe the cell morphology. Schaeffer-Fulton staining method was used to examine the presence of spore, and the bioMe'rieux Gram Stain kit according to the manufacturer's instructions (Beijing Land Bridge Technology Co., Ltd) was used to determine Gram reaction. All other physiological and biochemical tests were carried out based on those of Gordon et al. (Gordon, 1973) and have been described previously (Priest, 1988).

\section{Results}

\subsection{Screening result of active strain}

80 bacteria strains were isolated first from the soil samples by the Beef extract peptone medium plate at $25^{\circ} \mathrm{C}$. Only two strains, numbered C44 and D56, which can transform rutin to isoquercitrin, were obtained by TLC (Fig.1).

These two strains were re-screened by HPLC with rutin and isoquercitrin standards as control. Retained time of rutin and isoquercitrin were $5.277 \mathrm{~min}$ and $6.016 \mathrm{~min}$, respectively (Fig.2). It means that rutin and isoquercitrin could be separated effectively under the experiment condition. While the result HPLC indicated that strain C44 had a higher conversion ability than strain D56 (data not shown), which proved by an evident elution peak corresponding to isoquercitrin (Fig.2). Therefore, the further quantitative analysis was performed

Strain C44 was cultured in the rutin-Beef extract-peptone broth for $24 \mathrm{~h}$ to prepare the fermentation supernatant and suspension of cell precipitation for checking conversion activity. After the examination, isoquercitrin was only found in cell precipitation transfer system. It suggested that the active enzyme was intracellular enzyme.

Further quantitative analysis was performed with the standard curves manufactured by Excel2003. The regression equations of rutin and isoquercitrin were $y=12002 x+946.85\left(R^{2}=0.9977\right)$, and $y=19661 x+20.353\left(R^{2}=0.9998\right)$, respectively (Fig.3 and Fig.4). The figures showed that rutin and isoquercitrin had good linearities within the tested concentration range. After injected three times, the rutin transformation rate of strain $\mathrm{C} 44$ is $50.36 \%$ and the final isoquercitrin is $0.77 \mathrm{mg} / \mathrm{mL}$ by calculation.

\subsection{Identification result of strain C44}

Nearly complete 16S rDNA sequence of 1499 bp of strain C44 was obtained, and it was deposited in GenBank under the accession number of JQ345704. The 16S rDNA sequence was compared with the GenBank database. In the neighbour-joining phylogenetic tree, strain C44 and Bacillus litoralis SW-211 ${ }^{\mathrm{T}}$ clustered together with a high bootstrap value 
of $100 \%$ (Fig.5) sharing the similarity of $98.1 \%$.

Strain C44 is a rod-shaped, endospore-forming bacterial strain. Cells were $0.8 \sim 1.0 \mu \mathrm{m} \times 2.5 \sim 4.5 \mu \mathrm{m}$, in size, Gram-variable. Central, subterminal or terminal ellipsoidal endospores observed in swollen sporangia shows that their motile by means of peritrichous flagella. Colonies grown on nutrient agar are circular to slightly irregular, smooth, slightly raised, yellowish white and 2 3 $\mathrm{mm}$ in diameter after 2 days of incubation at $37^{\circ} \mathrm{C}$. Colony colour becomes dark from the centre as cultures age.

Optimal growth temperature for strain $\mathrm{C} 44$ is $37^{\circ} \mathrm{C}$. Optimal $\mathrm{pH}$ for growth is 7.5. Optimal growth occurs in the presence of $2-3 \%(w / v) ~ N a C l$, and growth does not occur without $\mathrm{NaCl}$ or in the presence of $>11 \%(w / v) ~ N a C l$. Anaerobic growth does not occur on MA or on MA supplemented with nitrate.

The strain is Catalase-positive and Urease-negative. Hypoxanthine, tyrosine and xanthine are not hydrolysed. $\mathrm{H}_{2} \mathrm{~S}$ and indole are not produced. Arginine dihydrolase, lysine decarboxylase, ornithine decarboxylase and tryptophan deaminase are absent. Acid is produced from L-arabinose, D-cellobiose, D-fructose, Dgalactose, D-melezitose, melibiose, D-raffinose, L-rhamnose, D-ribose, sucrose and D-trehalose.

All these phenotype characteristics serve to classify strain $\mathrm{C} 44$ as a member of the species Bacillus litoralis. Therefore strain C44 was identified as Bacillus litoralis on species level.

\section{Discussion}

According to our recent report, polyamide film TLC method only fit for first screening of quercetin-producing strains (Lu et al., 2012). In this research, we found that this TLC program also can separate isoquercitrin with rutin and quercetin quite well, so it was applied to screen the isoquercitrin producing strains preliminarily. And the potential active strains were also re-screened by HPLC determination further.

The result showed that the transformation rate of strain $\mathrm{C} 44$ from rutin to isoquercitrin is desirable. It suggests that this enzyme may be useful for industrial hydrolysis of rutin to isoquercitrin. While it may not meet the need of industrial production, this wild type bacterium will illustrate a good prospect of application after breeding and optimization of transformation conditions.

Many microorganisms have been reported for its ability of degrading rutin, such as Aspergihs flavus (Krishnamurty et al. 1970), Aspergillus niger (Shintaro et al. 1985), Penicillium rugulosum IFO 7242 (Tatsuya et al., 2000), Penicillum rugulosum (Narikawa et al., 2000), Actinoplanes missouriensis DSM 43046 ( Karsten R. et al., 2006), Thermoactinomyces vulgaris (Yang et al. 2009), Pyrococcus furiosus (Hyun-Koo Nam et al., 2012). The rutin degrade enzyme of Bacillus litoralis $\mathrm{C} 44$ is $\alpha$-rhamnosidase. Just like the reported enzymes, such as $\alpha$-L-rhamnosidase from Aspergillus niger (Shintaro et al. 1985), $\beta$-rutinosidase from Penicillium rugulosum IFO 7242 (Tatsuya et al., 2000), rutin- $\alpha$-rhanmosidase (Wang et al., 2004) and so on, all these enzymes have a remarkable specificity that the glycosidase did not hydrolyze any other substances, but only rutin. The enzyme in Bacillus litoralis $\mathrm{C} 44$ can specifically hydrolyze the end of the rhamnose glycosyl in many polysaccharose glucoside substances. So it has a highly specific of rutin, and the product is isoquercitrin only. Comparing with the reported rutin degrading microbes, this is the first time that Bacillus was found to be capable of transforming rutin to isoquercitrin.

\section{References}

[1] Kreft S., Knapp M., Kreft I. (1999). Extraction of rutin from buckwheat (Fagopyrum esculentum Moench) seeds and determination by capillary electrophoresis. J Agric Food Chem,47(11), 4649-4652. DOI: 10.1021/jf990186p

[2] Kuntic V., Pejic N., Ivkovic B. (2007). Isocratic RP-HPLC method for rutin determinationin solid oral dosage forms. J Pharm Biomed Anal,43(2),718-721. DOI:PMID: 16920326

[3] Cheng, X., Chen, X., Gao, Y., et al. (2004). Determined the contents of rutin and quercetin by RP-HPLC. Chinese Traditional Patent Medicine, 26(8), 680-682. DOI: cnki: ISSN: 1001-1528. 0.2004-08-028 
[4] Wang K., Yu H., Jin F.(2004). Purification and characterization of rutin- $\alpha$-rhanmosidase. Journal of Dal ian Institute of Light Industry, 23(1), 30-33. DOI: 1005-4014(2004) 01-0030-04

[5] Dolf De Rovira, Dictionary of Flavors(second edition), Iowa: Wiley-Blackwell, 2008, p371

[6] Shen, S., Chen, Y., Hsu, F.(2003). Differential apoptosis-inducing effect of quercetin and its glycosides in human promyeloleukemic HL-60 cells by alternative activation of the caspase 3 cascade. J. Cell. Biochem, 89(5): 1044-1055. DOI: 10.1002/jcb.10559

[7] Jin, Y. (2006). Structure-effect relationship of anti-free radical effects of quercetin and Its monoglycoside isoquercetin and diglycoside rutin. Dalian medical university, DOI:CNKI: CDMD: 2.2006.141375

[8] Jin, Y., Lü, Y., Han, G., et al. (2007). Comparative study on in vitro anti-free radical activities of quercetin, isoquercetin, and rutin. Chinese Traditional Patent Medicine, 38(3), 408-412. DOI: CNKI:ISSN:0253-2670.0.2007-03-032

[9] Zhang, R., Yao, Y., Wang, Y. (2011). Antidiabetic activity of isoquercetin in diabetic KK -Ay mice. Nutrition \& Metabolism, 8(85). DOI: 10.1186/1743-7075-8-85

[10] Yang X., Jiang Y., Li J. (1996). A new flavonol glucoside from aerial parts of Manaplant Hlhagi (Alhagi pseudoalhagi). Chinese Traditional and Herbal Drugs, 27(12),707-711

[11] Li, H.(2004). Studies on the synthesis of the 3-substituted quercetin. The Academy of Military Medical Sciences of the Chinese PLA. DOI:CNKI:CDMD:2.2004.097226

[12] Wang, J., Zhao, L., Sun, G. (2011). A comparison of acidic and enzymatic hydrolysis of rutin. Afr J Biotechnol,10(8),1460-1466. DOI: 10.5897/AJB10.1897

[13] H’elder Vila-Real, Ant'onio J. Alfaia, M. Ros'ario Bronze(2011). Enzymatic synthesis of the flavone glucosides, prunin and isoquercetin, and the aglycones, naringenin and quercetin, with selective $\alpha$-L-rhamnosidase and $\beta$-D-glucosidase activities of naringinase. SAGE-Hindawi Access to Research, Enzyme Research. 2011, 1-11. DOI: $10.4061 / 2011 / 692618$

[14] Karesten R., Susanne F.(2006). Identification of linear plasmid pAM1 in the flavonoid degrading strain Actinoplanes missouriensisT (DSM 43046). Plasmid, 55(33), 249-254

[15] Marmur, J. (1961). A procedure for the isolation of deoxyribonucleic acid from microorganisms. Journal of Molecular Biology, 3, 208-218.

[16]Lane, D.J. (1999). 16S/23S rDNA Sequencing. In: Nucleic acid techniques in bacterial systematics (eds Stackebrandt, E., Goodfellow, M.). John Wiley \& Sons, Chichester, pp115-175.

[17]Lu, Z., Liu, Z., Wang, L., et al. (2001). Saccharopolyspora falva sp. nov. and Saccharopolyspora thermopohila sp.nov., novel actinomycetes from soil. International Journal of Systematic and Evolutionary Microbiology, 51, 319-325.

[18] Hall, T.A. (1999). BioEdit, a user-friendly biological sequence alignment editor and analysis program for Windows 95/98/NT. Nucleic Acids Symp Ser, 41, 95-98.

[19] Saitou, N. \& Nei, M. (1987). The neighbor-joining method: a new method for reconstructing phylogenetic trees. Molecular Biology and Evolution, 4,406-425.

[20] Tamura, K., Peterson, D., Peterson, N., et al. (2011). MEGA5: Molecular evolutionary genetics analysis using maximum likelihood, evolutionary distance, and maximum parsimony methods. Molecular Biology and Evolution, 28, 2731-2739. DOI: 10.1093/molbev/msr121

[21] Gordon, R.E., Haynes, W.C. \& Pang, C. (1973). The genus Bacillus. Agriculture Handbook no. 427. United States Department of Agriculture, Washington, D. C.

[22] Priest, F, G., Goodfellow, M. \& Todd, C. (1988). A numerical classification of the genus Bacillus. Journal of Genetic Microbiology. 47, 1039-1046.

[23]Lu Z., Zhang Y., Shi N.(2012). Screening and identification of a quercetin-producing bacterium Paenibacillus glucanolyticu D3. Environment and Natural Resources Research, 2(1), 106-113. DOI:10.5539/enrr.v2n1p106 
[24] Krishnamurty, H.G. \& Simpson, F.J. (1970). Degradation of rutin by Aspergilis flavus. The Journal of Biological Chemistry, 245(6):1467-1471.

[25] Shintaro K., Sachiko E., Reiko T. (1985). Synthesis of some disaccharides containing an L-rhamnopyranosyl or L-mannopyranosyl residue, and the substrate-specificity of $\alpha$-L-rhamnosidase from Aspergillus niger . Agric. Biol. Chem., 49 (1), 55-62

[26] Tatsuya N., Hirofumi S., Takaaki F. (2000). A $\beta$-rutinosidase from Penicillium rugulosum IFO 7242 that is a paculiar flavonoid glycosidase. Biosci. Biotechnol. Biochem., 64(6), 1317-1319

[27] Narikawa, T., Shinoyama, H. \& Fujii, T. (2000). A $\beta$-rutinosidase from Penicillum rugulosum IFO 7242 that is a peculiar flavonoid glycosidase. Bioscience, Biotechonoly and Biochemistry. 64(6), 1317- 1319. DOI:PMID: 10923813

[28] Yang, C.H., Huang, Y.C., Chen C.Y. (2009). Degradation of rutin by Thermoactinomyces vulgaris and other thermophilic compost isolates. Journal of Agricutral and Food Chemistry. 57, 5095-5099. DOI:10.1021/jf900617z Hyun-Koo Nam, Seung-Hye Hong, Kyung-Chul Shin, Deok-Kun Oh(2012). Quercetin production from rutin by a thermostable b-rutinosidase from Pyrococcus furiosus. Biotechnol Lett, 34,483-489. DOI:10.1007/s10529-011-0786-2

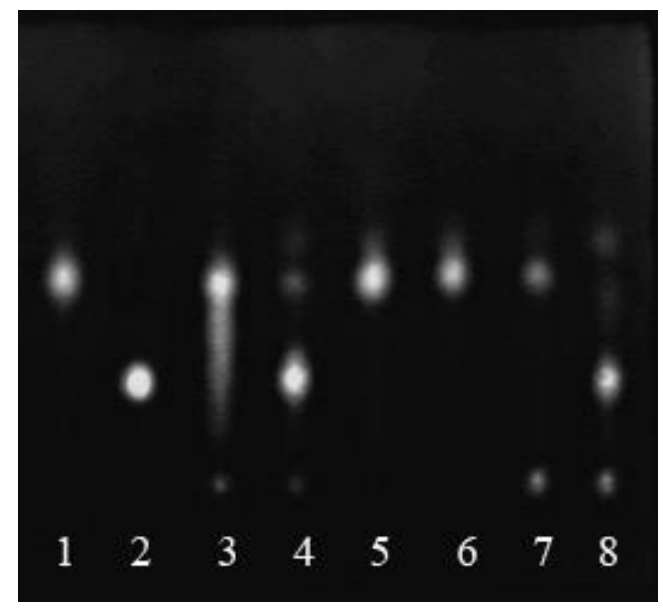

Fig 1. TLC determination result of some bacterial samples

1 rutin, 2 isoquercitrin, 3 negative control, 4-8 samples (5: strain C44; 8: strain D56)

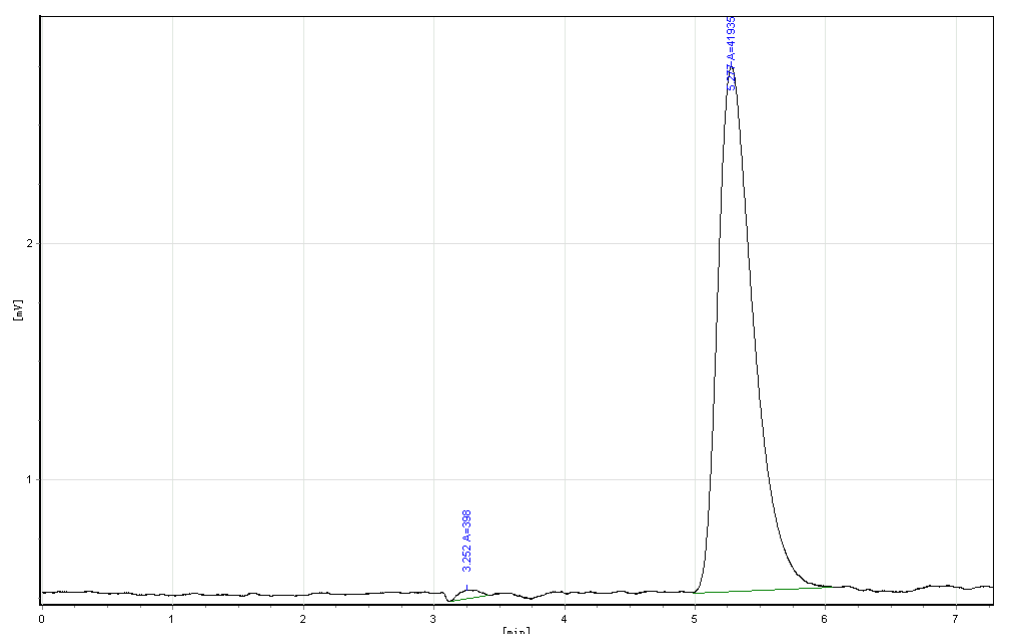

(a) 


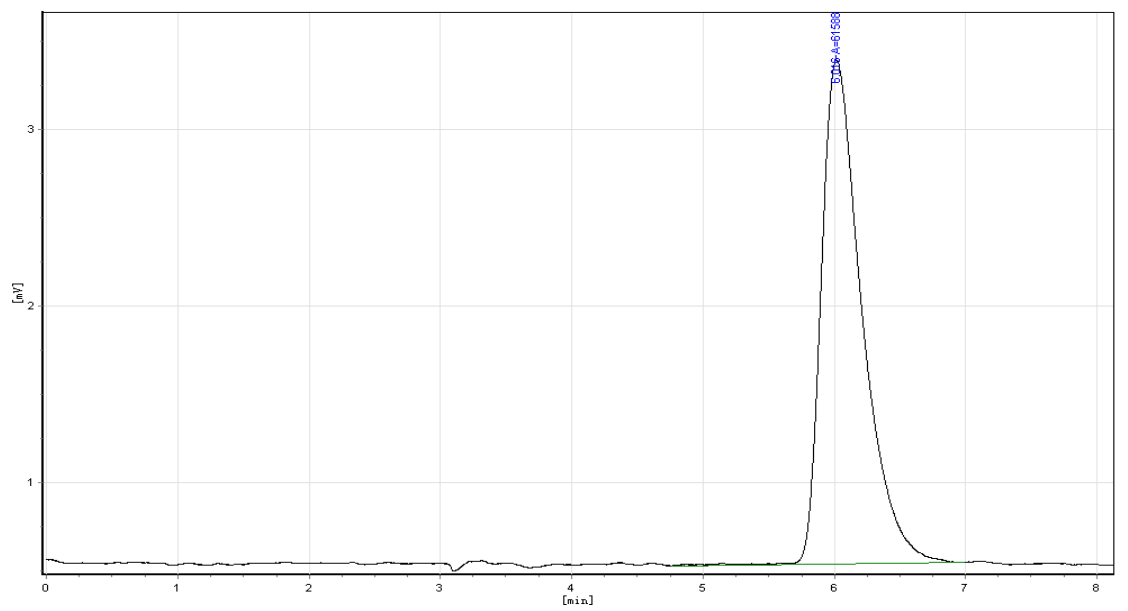

(b)

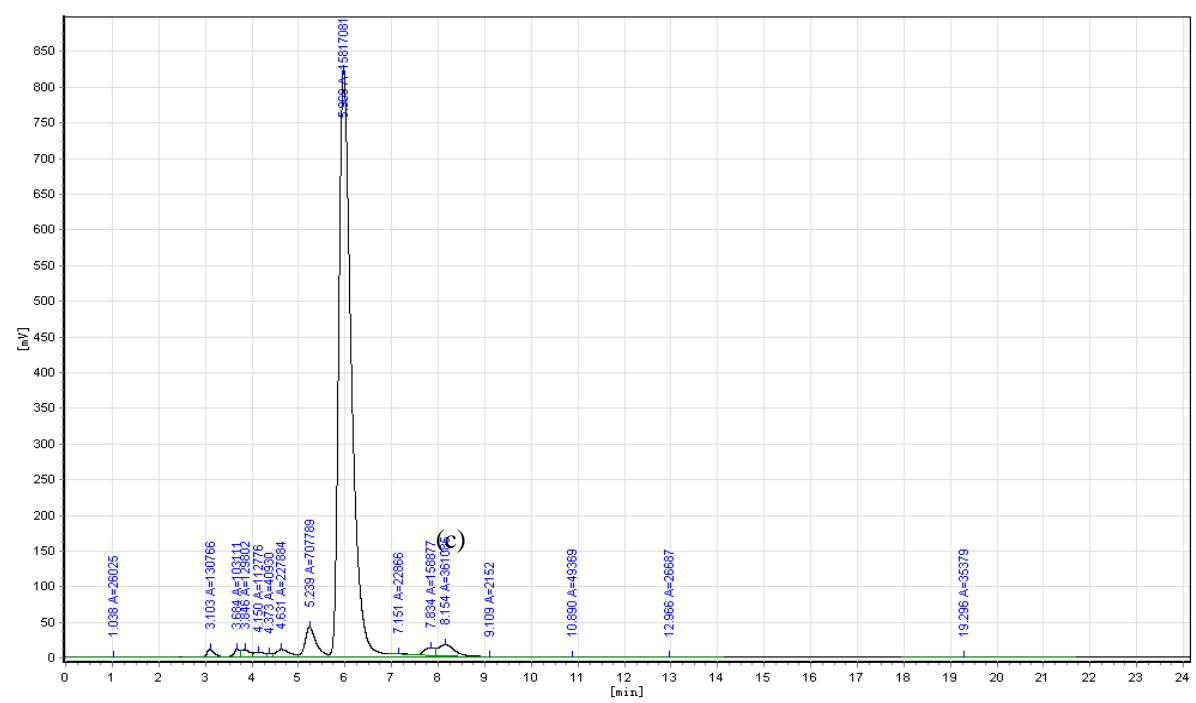

Fig 2. HPLC chromatograms of rutin standards and tested sample (a) rutin standard, (b) isoquercitrin standard, (c) tested sample of strain C44

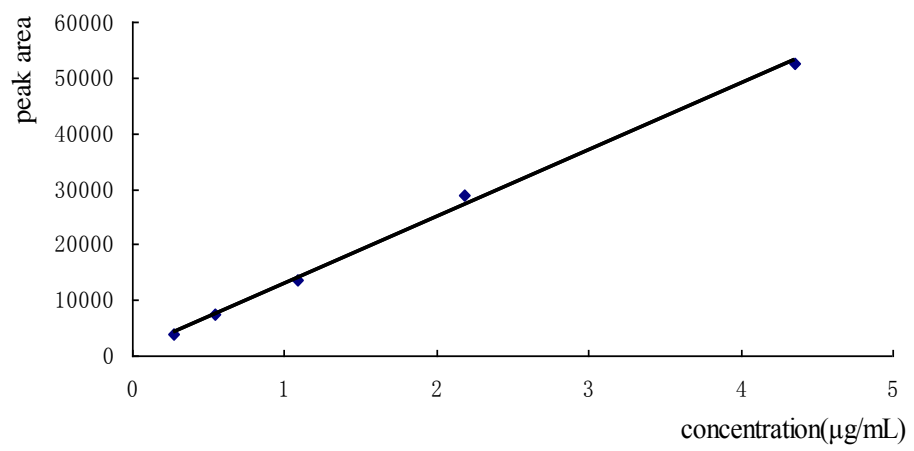

Fig.3 Standard curve of rutin by HPLC 


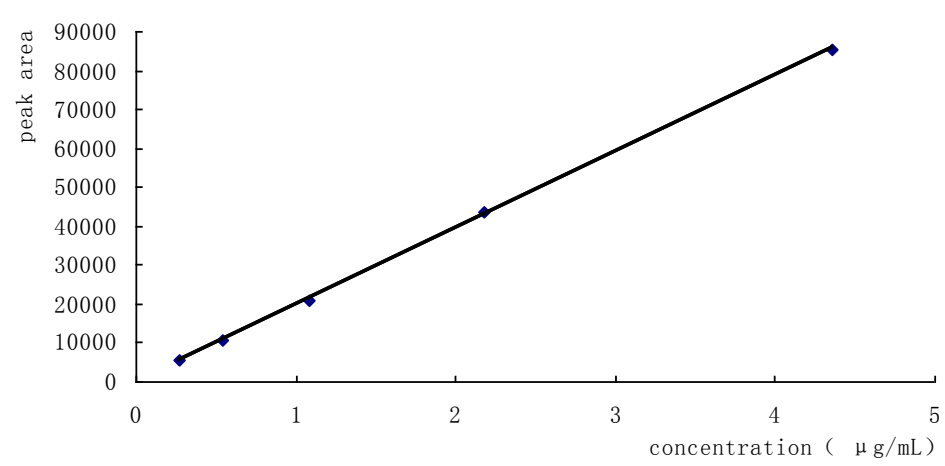

Fig 4. Standard curve of isoquercitrin by HPLC

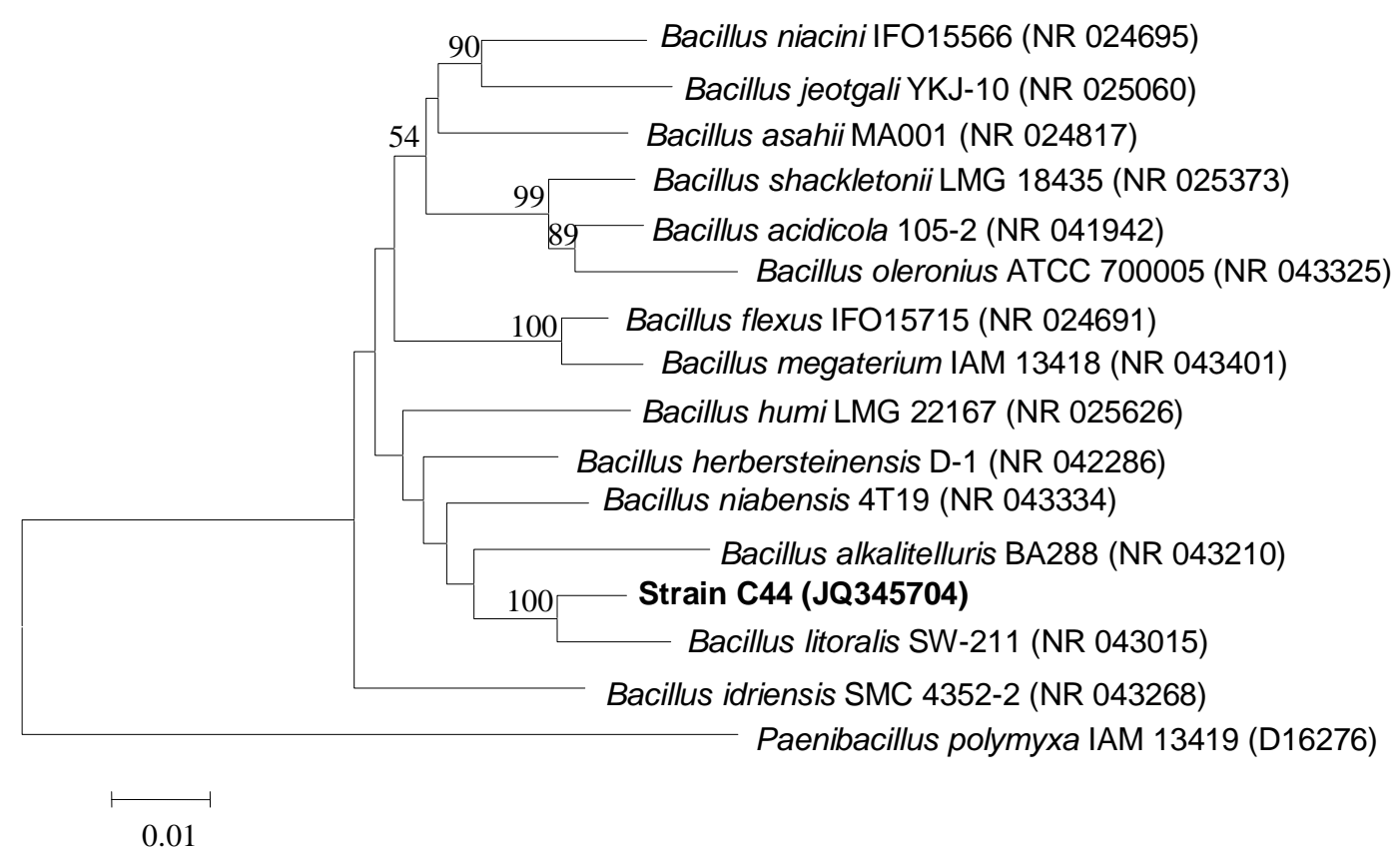

Fig 5. Neighbour-joining phylogenetic tree of strain C44 and related Bacillus representatives based on almost complete 16S rDNA sequences using Paenibacillus polymyxa (D16276) as outgroup 